\title{
ON RING EXTENSIONS FOR COMPLETELY PRIMARY NONCOMMUTATIVE RINGS
}

BY

\author{
E. H. FELLER AND E. W. SWOKOWSKI
}

0 . Introduction. It is the authors' purpose in this paper to initiate the study of ring extensions for completely $N$ primary noncommutative rings which satisfy the ascending chain condition for right ideals (A.C.C.). We begin here by showing that every completely $N$ primary ring $R$ with A.C.C. is properly contained in just such a ring. This is accomplished by first showing that $R[x], x$ an indeterminate where $a x=x a$ for all $a \in R$, is $N$ primary and then constructing the right quotient ring $Q(R[x])$. The details of these results appear in $\$ 1,7$ and 8 . The corresponding results for the commutative case are given by E. Snapper in [7] and [8].

If $R \subset A$, where $A$ is completely $N$ primary with A.C.C. then,from the discussion in the preceding paragraph, it would seem natural to examine the structure of $R(\sigma)$ when $\sigma \in A$ and $a \sigma=\sigma a$ for all $a \in R$ in the cases where $\sigma$ is algebraic or transcendental over $R$. These structures are determined in $\$ \$$ and 8 of the present paper.

The definitions and notations given in [2] will be used throughout this paper. As in [2], for a ring $R, N$ or $N(R)$ denotes the union of nilpotent ideals $\left({ }^{1}\right)$ of $R$, $P$ or $P(R)$ denotes the set of nilpotent elements of $R$ and $J$ or $J(R)$ the Jacobson radical of $R$. The letter $H$ is used for the natural homomorphism from $R$ to $R / N=\bar{R}$. If $B$ is a subset of $R$ then $\bar{B}$ denotes the image of $B$ under $H$. If $N=P$ in $R$ and if $R^{\prime}$ is a ring contained in $R$ then $N\left(R^{\prime}\right)=N \cap R^{\prime}$ and $R^{\prime}=R^{\prime} / N\left(R^{\prime}\right)$. Thus we consider the contraction of $H$ on $R^{\prime}$ as the natural homomorphism from $R^{\prime}$ onto $R^{\prime} / N\left(R^{\prime}\right)$.

Unlike the commutative case, the results of this paper will at times depend on the three conditions (i), (ii) and (iii) of $[2, \S 3]$. Therefore, we make the following definition.

DEFINITION 0.1. A ring $R$ with identity is called an extendable ring if it satisfies the three conditions:

(i) $P(q)$ is an ideal when $q$ is a right $P$ primary ideal $\left({ }^{2}\right)$.

(ii) $P(R)=N(R)$.

(iii) The nontrivial completely prime ideals of $R / N$ are maximal right ideals.

Received by the editors February 2, 1960 and, in revised form, September 11, 1961 and October 26, 1961.

(1) Ideal shall always mean two-sided ideal.

(2) See $[2, \S 1]$ for the meaning of $P(q)$. 
The authors wish to thank the referee for many pertinent and constructive comments.

1. Properties of $R[x]$. In [2] we defined a ring $R$ to be $N$ primary if $a b=0$, $a \neq 0$ implies $b \in N$ and $b \neq 0$ implies $a \in N$. A ring $R$ with identity is completely $N$ primary if $R / N$ is a division ring. Note that if $R$ is $N$ primary or completely $N$ primary then $N$ is a completely prime ideal and $N=P$. For the rings considered in this paper $N$ and $P$ shall always be equal.

THEOREM 1.1. If $R$ is $N$ primary then, in the polynomial ring $R[x], N[x]=P[x]$ $=N(R[x])=P(R[x])=J(R[x])$.

Proof. We may write $R[x] / N[x] \cong(R / N)[x]$ where $N[x] \subseteq J(R[x])$. Since $R / N$ is an integral domain it contains no nonzero nil ideals and hence, from Theorem 4 of $[6$, p. 12], $R[x] / N[x]$ is semisimple. Thus $J(R[x])=N[x]$.

Certainly $N[x]=P[x] \subseteq P(R[x])$. We now show that $P(R[x]) \subseteq P[x]$. If $f \in P(R[x])$ then $f^{n}=0$ for some positive integer $n$. Hence in $R[x] / P[x] \cong(R / P)[x]$ we have $(\tilde{f})^{n}=\tilde{0}\left({ }^{3}\right)$. Since $(R / P)[x]$ has no divisors of zero it follows that $f \in P[x]$.

Next we show that $N(R[x])=N[x]$. Clearly $N(R[x]) \subseteq P(R[x])=N[x]$. If $f \in N[x]$ then $f=a_{n} x^{n}+\ldots+a_{1} x+a_{0}$ for $a_{i} \in q_{i}$ where $q_{i}$ is an ideal of $R$ and $q_{i}^{t_{i}}=0$ for positive integers $t_{i}, i=0,1, \ldots, n$. Thus $f$ is contained in the ideal $k=q_{0}[x]+q_{1}[x]+\ldots+q_{n}[x]$ and $k^{t_{0}+t_{1}+\cdots+t_{n}+1}=0$. Hence $f \in N(R[x])$. This completes the proof.

As a consequence of this theorem we have, for an $N$ primary ring $R$, that $R[x] / N(R[x])=R[x] / N[x] \cong(R / N)[x]$. Thus we can consider $(R[x])^{-}$as $\bar{R}[x]$. In this case, the natural homomorphism $H$ from $R[x]$ onto $\bar{R}[x]$ maps the polynomial $\sum a_{i} x^{i}$ on $\sum \bar{a}_{i} x^{i}$.

THEOREM 1.2. Let $R$ be an $N$ primary ring. Then $f=a_{n} x^{n}+\ldots+a_{0}$ is $a$ unit of $R[x]$ if and only if $a_{0}$ is a unit of $R$ and $a_{1}, \ldots, a_{n} \in N$.

Proof. If $f$ is of this form then $\bar{f}$ is a unit of $\bar{R}[x]$. Hence by $2 \mathrm{a}$ of $[2] f$ is a unit of $R[x]$.

Conversely, if $f=a_{n} x^{n}+\ldots+a_{0}$ is a unit of $R[x]$ then $\bar{f}=\bar{a}_{n} x^{n}+\ldots+\bar{a}_{0}$ is a unit of $\bar{R}[x]$. But since $\bar{R}$ is an integral domain it follows that $\bar{f}=\bar{a}_{0}$ is a unit of $\bar{R}$ and $\tilde{a}_{1}=\ldots=\tilde{a}_{n}=\overline{0}$. Thus $a_{0}$ is a unit of $R$ and $a_{1}, \ldots, a_{n} \in N(R)$.

THEOREM 1.3. If $R$ is completely $N$ primary and $N^{n}=0$ for some positive integer $n$, then $R[x]$ is $N[x]$ primary.

Proof. This proof will be by induction on the smallest integer $n$ such that $N^{n}=0$. If $n=1$ obviously $R[x]$ is $N(R[x])$ primary. By Theorem $1.1 N(R[x])$ $=N[x]$ and thus $R[x]$ is $N[x]$ primary.

(3) Here the symbol $\sim$ denotes the coset modulo $P[x]$. 
Suppose the theorem is true for all rings $R$ where $N^{n}=0, N^{n-1} \neq 0$. Let $R$ satisfy the conditions of the theorem and $N^{n+1}=0, N^{n} \neq 0$. Consider the ring $R / N^{n}$. The radical of this ring is $N / N^{n}$ and $\left(N / N^{n}\right)^{n}$ is the zero coset. In addition since $\left(R / N^{n}\right) /\left(N / N^{n}\right) \cong R / N$ we have that $R / N^{n}$ is completely $N / N^{n}$ primary. Hence, by the induction hypothesis $\left(R / N^{n}\right)[x]$ is $\left(N / N^{n}\right)[x]$ primary.

Suppose in $R[x]$ we have $f g=0, g \neq 0$ and $f \notin N[x]$. Since $(R / N)[x]$ is an integral domain $g \in N[x]$. If $g \notin N^{n}[x]$, then $\tilde{f} \tilde{g}=\tilde{0}$ in $\left(R / N^{n}\right)[x]$ and from above $f \in\left(N / N^{n}\right)[x]$. Thus $f \in N[x]$, a contradiction. Suppose $g \in N^{n}[x]$. Let $a_{i}$ be the coefficient of the highest power of $x$ in $f$ which is not in $N$. Since $N^{n+1}=0$ and $f g=0$, we have $a_{i} b_{s}=0$ where $g=b_{s} x^{s}+\ldots+b_{0}, b_{s} \neq 0$. Since $R$ is completely $N$ primary $a_{i} \in N$. Thus in any case the assumption that $f g=0, g \neq 0, f \notin N[x]$ leads to a contradiction. Hence if $g \neq 0$ we can only conclude that $f \in N[x]$. Similarly if $g f=0$ and $f \neq 0$ then $g \in N[x]$. Therefore, $R[x]$ is $N[x]$ primary and the proof by induction is complete.

2. Extensions of rings. A ring $A$ is an extension of a ring $R$ if $R \subseteq A$. In the remainder of this paper, unless otherwise stated, we assume that if $A$ is an extension of $R$ then $R$ and $A$ have the same identity element. If $R \subseteq A$ then $A$ can be considered as an $R$ module $\left({ }^{4}\right)$ with submodule $R$. Thus, in order to develop a theory for extensions of a ring, it is convenient to discuss some notions concerning modules.

Let $R$ be a ring with identity and $M$ a unital $R$ module. If $R_{1}$ and $M_{1}$ are nonempty subsets of $R$ and $M$ respectively, then $R_{1} M_{1}$ denotes the set of all finite sums $\sum r_{i} m_{i}$ where $r_{i} \in R_{i}$ and $m_{i} \in M_{1}$. A subset $S$ of $M$ is called a generating system of a submodule $Q$ of $M$ if $Q=R S$. If $S$ contains a finite number of elements then $Q$ is said to be finitely generated. A finite generating system $S$ of $Q$ is called a basis of $Q$ if $Q$ does not have a generating system containing fewer elements than $S$. If $Q$ is finitely generated then the $\operatorname{rank} \rho(Q)$ of $Q$ is the number of elements in a basis of $Q$.

The following theorem was proved in $[7, \mathrm{p} .685]$ for commutative rings. The proof carries over immediately to the noncommutative case.

THEOREM 2.1. Let $M$ be an $R$ module and $Q$ a submodule of $M$ such that $M-Q$ is finitely generated. If $q$ is any $J$ ideal of $R$ and $Q^{\prime}$ any submodule of $M$ let $\widetilde{S}$ denote the image of the subset $S$ of $M$ under the natural homomorphism from $M$ onto $M-q Q^{\prime}$. Then $M=Q+R S$ if and only if $\tilde{M}=\tilde{Q}+R \tilde{S}$. Consequently $\rho(M-Q)=\rho(\tilde{M}-\tilde{Q})$ and if $\tilde{M}=\tilde{Q}$ then $M=Q$.

If a ring $A$ has finite rank as a module over a subring $R$ we call the rank the degree $[A: R]$ of the ring extension and say that $A$ is a finite extension of $R$.

(4) $R$ module shall always mean left $R$ module. For a discussion of left $R$ modules read Chapter I of [6]. 
THEOREM 2.2. Let $R$ and $A$ be rings where $R \subseteq A$ and suppose every right unit of $A$ is a left unit. Then $R=A$ if and only if $[A: R]=1$.

Proof. If $R=A$ then the identity element of $A$ is a basis of $A$ and hence $[A: R]$ $=1$. Conversely if $[A: R]=1$, let $z$ be a basis of $A$. Then since $1 \in A$ we have $a z=1$ for some $a \in R$. Thus $z$ is a right and left unit of $A$ and certainly regular. Then for $z^{2} \in A$ we have $b z=z^{2}$ for some $b \in R$ which implies that $b=z \in R$. Thus $R=A$.

By statements 1.1 and 1.2 of [2] it follows that the conditions of Theorem 2.2 are satisfied when $A$ is a $J$ primary ring. In addition, if $R$ and $A$ have the same identity element and $A / N$ is a principal ideal domain, then by the discussion in $\S 5$ of [2] we know that Theorem 2.2 is valid.

If $R$ and $A$ are rings where $R \subseteq A$, the contraction $q_{*}$ of an ideal $q$ of $A$ is defined as the largest ideal of $R$ which is contained in $q$, i.e., $q_{*}=q \cap R$. The extension $q^{*}$ of an ideal $q$ of $R$ in $A$ is defined as the smallest ideal of $A$ which contains $q$, i.e., $q=A q A$, the set of all sums $\sum a_{i} q_{i} b_{i}$ where $q_{i} \in q$ and $a_{i}, b_{i} \in A$. Thus, by definition, $N(R)^{*}=A N(R) A$. Of particular importance to us is the case when $N(R)^{*}=N(R) A$ and in addition $N(R)^{*}=N(A)$. We make the following

Definition 2.1. A ring $A$ is called a principal extension of a subring $R$ if $N(A)=N(R)^{*}=N(R) A$.

If $R$ is $N$ primary then, by Theorem 1.1 , the ring $R[x]$ is a principal extension of $R$.

THEOREM 2.3. Let $A$ be a finite principal extension of $R$. If $S$ is a subset of $A$ then $A=R S$ if and only if $\bar{A}=\bar{R} S$. Hence $[A: R]=[A: R]$ and if $R=A$ then $R=A$.

Proof. If $A$ is a principal extension of $R$ then $N(A)=N(R) A$. Consider $A$ as an $R$ module with $N(R) \subseteq J(R)$. The first part of the theorem then follows from Theorem 2.1 by setting $A=Q^{\prime}=M, Q=0$ and $q=N(R)$. If in Theorem 2.1 we let $A=Q^{\prime}=M, Q=R$ and $q=N(R)$ it follows that $\bar{R}=\bar{A}$ implies $R=A$.

TheOREM 2.4. Let $R=A_{0} \subseteq A_{1} \subseteq \ldots \subseteq A_{n}$, where $A_{i}$ is a finite principal extension of $A_{i-1}$ of degree $\left[A_{i}: A_{i-1}\right]=r_{i}$ for $i=1,2, \ldots, n$. Then, if all the rings $R, A_{1}, \ldots, A_{n-1}$ are completely $N$ primary, $\left[A_{n}: R\right]=r_{1} r_{2} \ldots r_{n}$.

Proof. Since $N\left(A_{n}\right)=N(R) A_{1} A_{2} \ldots A_{n}=N(R) A_{n}$ it follows that $A_{n}$ is a finite principal extension of $R$. Hence $\left[A_{n}: R\right]=\left[A_{n}: R\right]$. Since $A_{i}$ is a finite principal extension of $A_{i-1}, r_{i}=\left[\bar{A}_{i}: A_{i-1}\right]$. Consequently, since $\bar{R}, \bar{A}_{1}, \ldots, \bar{A}_{n-1}$ are division rings we have $\left[A_{n}: R\right]=r_{1} r_{2} \ldots r_{n}$.

\section{Degrees of ideals.}

Definition 3.1. Let $R$ and $A$ be rings where $R \subseteq A$. An ideal $p$ of $A$ has finite degree $\operatorname{deg}(p)$ if the ring $A / p$ is a finite extension of $R / p_{*}$. If $p$ has finite degree then $\operatorname{deg}(p)=\left[A / p: R / p_{*}\right]$. 
Definition 3.1 is equivalent to saying that if the rank of the $R$ module $A-p$ is finite then $\operatorname{deg}(p)=\rho(A-p)$.

THEOREM 3.1. Let $A$ be a principal extension of $R$ and $p$ an ideal of $A$ of finite degree. If $S$ is a subset of $A$ then $A=p+R S$ if and only if $\bar{A}=\bar{p}+\bar{R} S$. Hence $\operatorname{deg}(p)=\operatorname{deg}(\bar{p})$.

Proof. Apply Theorem 2.1 with $M=Q^{\prime}=A, Q=p$ and $q=N(R)$.

The following two results are proved in [7] for commutative rings. Using the definitions and results listed above the proofs now carry over, without essential modification, to the noncommutative case.

StATEMENT 3.1. An ideal $p$ of $R[x]$ has finite degree if and only if $p$ contains a monic polynomial $\left({ }^{5}\right)$.

THEOREM 3.2. If $R$ is $N$ primary, then an ideal $p$ of $R[x]$ has finite degree if and only if $\bar{p}$ has finite degree in $R[x]$. In this case if $B$ is a subset of $R[x]$ then $R[x]=R B+p$ if and only if $\bar{R}[x]=\bar{R} \bar{B}+\bar{p}$. Consequently $\operatorname{deg}(p)=\operatorname{deg}(\bar{p})$.

Notice that if $\bar{R}$ is a division ring then $\bar{R}[x]$ is a principal ideal domain and if $\bar{p}=(\bar{f})$ then $\operatorname{deg} \bar{p}=D(\bar{f})$. (The symbol $D(f)$ denotes the degree of the polynomial $f$.)

DEFINITION 3.2. The order of a regular polynomial $f$ of $R[x]$ is the minimal degree of the nonzero polynomials of $f R[x]$. The order of $f$ is denoted by $O(f)$.

Note that $O(f)=D(f)$ when $R$ is an integral domain.

LEMMA 3.1. If $R$ is completely $N$ primary and $N^{n}=0$ for a positive integer $n$, then $O(f)$ is equal to the exponent of the highest power of $x$ in $f$ whose coeffcient is a unit of $R$.

Proof. Let $f=a_{l} x^{l}+\ldots+a_{m} x^{m}+\ldots+a_{0}$ where $a_{l}, \ldots, a_{m+1} \in N$ and $a_{m} \notin N$. Certainly $O(f) \leqq m$. If $N=0$, the theorem is obviously true. Assume inductively that the theorem is true for rings $R$ where $N^{r}=0, r \leqq n$. We shall show that the theorem is true for rings $R$ where $N^{n} \neq 0$ and $N^{n+1}=0$. Suppose $D(f g)<m$ where $g=b_{s} x^{s}+\ldots+b_{0}, b_{s} \neq 0$. Since $(R / N)[x]$ is an integral domain, we have $g \in N[x]$. Since $R$ is completely $N$ primary, $R / N^{n}$ is completely $N / N^{n}$ primary. Also, by Theorem 1.3, the coset of $f$ is regular in $R / N^{n}[x]$. If $g \in N[x]$ but $g \notin N^{n}[x]$ then, by the induction hypothesis, $D(f g)$ is not less than $m$. If, on the other hand, $g \in N^{n}[x]$ and $D(f g)<m$ then $a_{m} b_{s}=0$ which is impossible because $a_{m}$ is a unit of $R$. Thus, in any case, the assumption $D(f g)<m$ leads to a contradiction. Consequently $O(f)=m$.

THEOREM 3.3. Let $R$ be a completely $N$ primary ring with $N^{n}=0$ for some positive integer $n$. If, for $f \in R[x]$, the principal ideal $(f)=f R[x]=R[x] f$ then $(f)$ is generated by a monic polynomial of degree $m$ if an only if $f=a_{l} x^{l}$ $+\ldots+a_{m} x^{m}+\ldots+a_{0}$ where $a_{l}, \ldots, a_{m+1} \in N$ and $a_{m}$ is $a$ unit of $R$.

(5) A nonzero polynomial of $R[x]$ is called monic if its leading coefficient is a unit element of $R$. 
Proof. If $f$ is of this form let $B$ denote the set of elements $x^{m-1}, \ldots, x, 1$ of $R[x]$. Then we may write $\bar{R}[x]=\bar{R} \bar{B}+(\bar{f})$. From Theorem 3.2 it follows that $R[x]$ $=R B+(f)$. Thus $x^{m}=-b_{m-1} x^{m-1}-\ldots-b_{0}+f_{1}$ where $f_{1} \in(f)$ and $b_{i} \in R$, $i=0,1, \ldots, m-1$. Hence $g=x^{m}+b_{m-1} x^{m-1}+\ldots+b_{0} \in(f)$. Now there exist polynomials $h$ and $k$ such that $f=h g+k$ where $k=0$ or $D(k)<m$. By Lemma 3.1, $O(f)=m$ and hence $k=0$. Thus $R[x] g=(f)$. Similarly $g R[x]=(f)$. Thus $(g)=(f)$ where $g$ is monic.

Conversely, let $(f)=(g)$ where $g$ is monic of degree $m$. Then $O(f)=O(g)$ (by the definition of $O()$ ), while $O(g)=m$ by Lemma 3.1 (because $g$ is monic). Thus $O(f)=m$, which means again by Lemma 3.1 that $f$ is of the required form.

4. Primary ideals in $R$, where $R / N$ is a principal ideal domain. If $R$ is a principal ideal domain then the A.C.C. holds for right ideals and hence $P(q)$ is an ideal when $q$ is a right $P$ primary ideal of $R$. Moreover, in this case $P=N=0$ and the completely prime ideals of $R / N$ are maximal right ideals. Hence, by Definition 0.1, a principal ideal domain is an extendable ring.

If $q$ is a $P$ primary ideal then $P(q)=N(q)$ since $P(q)$ is a completely prime ideal. Thus $P(q)$ is also a maximal left ideal.

THEOREM 4.1. If $R$ is a principal ideal domain and $q=(a)$ is a $P$ primary ideal in $R$ with $P(q)=(b)$ then $b$ is irreducible and $a=v b^{n}=b^{n} u$ where $u$ and $v$ are units of $R$.

Proof. If $b=c d$ then $c$ or $d$ must be in (b). Suppose $d \in(b)$, say $d=e b\left({ }^{6}\right)$. Then $b=c e b$ and $c$ is a unit of $R$. If $c \in(b)$, say $c=b e$, then $b=b e d$ and $d$ is a unit of $R$. Hence $b$ is irreducible.

For the second part of the proof we have $(a) \subseteq(b)$ where $b$ is irreducible. Let $a=b c$ where $c \in R$. If $b \notin(a)$ then $c^{n} \in(a)$ since $(a)$ is $P$ primary. Hence $c \in P((a))=(b)$. Thus $c=b d$ and $a=b^{2} d$. This process continues until $a=b^{n} d^{\prime}$ where $b^{n} \in(a)$ and $b^{n-1} \notin(a)$. Consequently, $b^{n}=a e$ and $a=a e d^{\prime}$. Thus $d^{\prime}$ is a unit and $a=b^{n} v=u b^{n}$ where $u$ and $v$ are units of $R$.

LEMMA 4.1. If $R / N$ is a principal ideal domain and $q$ is an ideal of $R$, then there is an element $a$ in $q$ such that $q=a R+N^{\prime}=R a+N^{\prime}$ where $N^{\prime}=q \cap N$.

Proof. Let $N^{\prime}=q \cap N$. Since $\bar{R}$ is a principal ideal domain $\bar{q}=(\bar{a})=\bar{a} \bar{R}=\bar{R} \bar{a}$ for some $\bar{a} \in \bar{R}$. Let $a$ be any element of $q$ such that $a H=\bar{a}$. Obviously $a R+N^{\prime} \subseteq q$. Moreover, if $b \in q$ then $b H=\bar{a} \bar{r}$ where $\bar{r} \in \bar{R}$. Thus $b=a r+n$ where $r \in R$ and $n \in N$. Since $n=b-a r, n$ is also in $q$ and therefore $n \in N^{\prime}$. Hence $q=a R+N^{\prime}$. Similarly $q=R a+N^{\prime}$.

THEOREM 4.2. Let $R$ be an extendable ring such that $R / N$ is a principal ideal domain. If $q$ is a $P$ primary, not nil, nontrivial ideal of $R$ then

$$
q=\left(v \pi^{k}+n\right) R+N^{\prime}=R\left(v \pi^{k}+n\right)+N^{\prime}
$$

(6) Note that if $b$ is any element of a principal ideal domain $R$ such that $b R$ is a left, whence two-sided ideal, then from [5, p. 37] $b R=R b$, which one may denote by $(b)$. 
where $v$ is a unit of $R, n \in N, \pi$ is an irreducible element of $R$ and $N^{\prime}=N \cap q$. Moreover, $P(q)=\pi R+N$ and $R / P(q)$ is a division ring.

Proof. By Lemma 4.1, $q=a R+N^{\prime}=R a+N^{\prime}$ where $a \in q$. From $3 \mathrm{~b}$ of [2] the not nil ideal $a R+N^{\prime}$ is $P$ primary in $R$ if and only if the ideal $(\bar{a})$ is $P$ primary in $\bar{R}$. From Theorem 4.1 we may write $\bar{a}=\bar{v} \bar{\pi}^{k}=\bar{\pi}^{k} \bar{u}$ where $\bar{\pi}$ is irreducible in $\bar{R}$ and $\bar{u}, \bar{v}$ are units of $\bar{R}$. Hence $a=\pi^{k} u+n_{1}=v \pi^{k}+n_{2}$ where $u$ and $v$ are units of $R$ and $n_{1}, n_{2} \in N$.

To find $P(q)$, write $\bar{q}=\left(\bar{v} \bar{\pi}^{k}\right)=\left(\bar{\pi}^{k}\right)=\bar{\pi}^{k} \bar{R}$. Clearly $\bar{\pi} \bar{R} \subseteq P(\bar{q})$. Moreover, since $\bar{\pi}$ is irreducible, the ideal $\bar{\pi} \bar{R}$ is maximal in $\bar{R}$ and hence $P(\bar{q})=\bar{\pi} \bar{R}$. From $2 \mathrm{~g}$ of [2] we have $(P(q))^{-}=(\pi R)^{-}$and hence $P(q)=\pi R+N$. The fact that $R / P(q)$ is a division ring is a consequence of statement 3.1 of [2].

THEOREM 4.3. If $R$ is a completely $N$ primary ring which satisfies the A.C.C. for right ideals then $R[x]$ is an extendable ring, $R[x]$ is a principal ideal domain and $R[x]$ is $N[x]$ primary.

Proof. If $R$ satisfies the A.C.C. then $N$ is nilpotent and, from Theorem 1.3, $R[x]$ is $N[x]$ primary. Since $\bar{R}$ is a division ring, $R[x]$ is a principal ideal domain $\left({ }^{7}\right)$. It remains to show that $R[x]$ is an extendable ring. If $q$ is a right $P$ primary ideal in $R[x]$ then, since $R[x]$ satisfies the A.C.C., $P(q)$ is an ideal of $R[x]$ and, from Theorem 1.1, $P(R[x])=N(R[x])$. Finally, condition (iii) of Definition 0.1 holds since $R[x] / N(R[x])=R[x] / N[x]=R[x]$ is a principal ideal domain.

5. $R[x]$, where $R$ is a completely $N$ primary ring. In this section let $R$ denote a completely $N$ primary ring which satisfies the A.C.C. for right ideals. By Theorem 4.3, $R[x]$ is an extendable ring, $N(R[x])=N[x]$ and $(R / N)[x]$ is a principal ideal domain. In addition, by $\left[6\right.$, p. 199], $(N[x])^{t}=0$ for some integer $t$.

Certainly the not nil ideals of $R[x]$ are the regular ideals of $R[x]\left({ }^{8}\right)$. If $q$ is a regular ideal in $R[x]$ then, by $\S 4, \bar{q}=(\bar{f})$ in $R[x]$ where $f$ is regular in $R[x]$. Hence all the regular ideals of $R[x]$ are of the form $q=f R[x]+N^{\prime}$ where $f$ is regular in $R[x]$ and $N^{\prime}=q \cap N[x]$. By $\S 3$ we have $O(f)=D(\bar{f})$.

THEOREM 5.1. An ideal $q$ of $R[x]$ has finite degree if and only if $q$ is a regular ideal. In this case, $\operatorname{deg}(q)=O(\tilde{f})=D(\tilde{f})$.

Proof. By Theorem 3.2, $q$ has finite degree if and only if $\bar{q}$ has finite degree, i.e., if and only if $q \nsubseteq N[x]$. Thus a necessary and sufficient condition that $q$ have finite degree is that $q$ be regular. Again, by Theorem 3.2, the degree of $q$ is the same as the degree of $\bar{q}=(\bar{f})$. Since $\bar{R}$ is a division ring $D(\bar{f})=O(\bar{f})$.

(7) See [5, Chapter 3].

(8) An ideal $q$ of a ring $R$ is called regular if it contains at least one regular element. Thus if $R$ is $N$ primary then it contains only regular ideals and nil ideals, for if an ideal $q$ is not regular then every element of $q$ is a divisor of zero and hence $q \subseteq N$. 
If $q$ is a regular, nontrivial $N$ primary ideal of $R[x]$, then by Theorem 4.2, $q=\left(v(x) p(x)^{k}+n(x)\right) R[x]+N^{\prime}$ where $v(x)$ is a unit of $R[x], \bar{p}(x)$ is an irreducible polynomial of $\bar{R}[x], n(x) \in N[x]$ and $N^{\prime}=N[x] \cap q$. The radical $N(q)$ $=p(x) R[x]+N[x]$. Then $\operatorname{deg}(q)=k \operatorname{deg}(\bar{p})$ and $\bar{R}[x] /(\bar{p}(x))$ is a division ring. Thus $R[x] / q$ is a completely $N$ primary ring.

6. Simple algebraic extensions. In this section let $R \subseteq A$ where $R$ and $A$ are completely $N$ primary rings which satisfy the A.C.C. If $\sigma \in A$, where $a \sigma=\sigma a$ for all $a \in R$, the symbol $R[\sigma]$ shall denote the smallest subring of $A$ containing $R$ and $\sigma$. The symbol $R(\sigma)$ shall denote the smallest completely $N$ primary ring containing $R$ and $\sigma$. In the latter case, $R(\sigma)$ is called a simple extension of $R$. Certainly $R[\sigma] \subseteq R(\sigma)$ and, if $x$ is an indeterminate, $R[\sigma]$ is the homomorphic image of the polynomial ring $R[x]$ under the homomorphism $f(x) \rightarrow f(\sigma)$. Since $R[\sigma]$ is a subring of a completely $N$ primary ring we know that $R[\sigma]$ is $P$ primary. The kernel of the homomorphism must then be a $P$ primary ideal $q$ of $R[x]$ and $R[x] / q \cong R[\sigma]$. In addition $q_{*}=q \cap R$ is the zero ideal since $q$ is the set of polynomials which have $\sigma$ as a root. As in $\S 5, q$ is either a regular ideal or a nil ideal.

Definition 6.1. Let $R \subseteq A$ and let $\sigma \in A$ where $a \sigma=\sigma a$ for all $a \in R$. If $\sigma$ satisfies at least one regular polynomial of $R[x]$ then $\sigma$ is called central algebraic with respect to $R$. If $\sigma$ satisfies only nilpotent polynomials of $R[x]$ then $\sigma$ is called central transcendental with respect to $R\left({ }^{9}\right)$. The ideal $q$ consisting of the polynomials of $R[x]$ which have $\sigma$ as a root is called the defining ideal of $\sigma$.

We call $R(\sigma)$ a simple algebraic extension of $R$ if $\sigma$ is algebraic with respect to $R$ and a simple transcendental extension of $R$ if $\sigma$ is transcendental with respect to $R$.

Let $S=R(\sigma)$ be a simple algebraic extension of $R$ and let $q$ be the defining ideal of $\sigma$. Then $q$ is a not nil, nontrivial $P$ primary ideal of $R[x]$. By $\S \S 4$ and 5 we may write $q=\left(v(x) p(x)^{k}+n(x)\right) R[x]+N^{\prime}$ where the symbols have the same meanings as before. Then $R[x] / q$ is a completely $N$ primary ring whose residue class ring is isomorphic to $\bar{R}[x] /(\bar{p}(x))$ and $R[x] / q$ is an extension of degree $k D(\bar{p}(x))$ of $R / q_{*}=R$. Since $R[x] / q \cong R[\sigma]$ and $R[\sigma]$ satisfies the A.C.C. we have

THEOREM 6.1. If $S=R(\sigma)$ is a simple algebraic extension of $R$, the defining ideal $q$ of $\sigma$ has the form $q=\left(v(x) p(x)^{k}+n(x)\right) R[x]+N^{\prime}$ where $v(x)$ is a unit of $R[x], \bar{p}(x)$ is irreducible in $\bar{R}[x], n(x) \in N[x]$ and $N^{\prime}=q \cap N[x]$. Then $S=R(\sigma)=R[\sigma]$ which satisfies the A.C.C. Moreover, $S$ is a finite extension of $R$ where $[S: R]=k D(\bar{p}(x))$. The division ring $S=\bar{R}(\bar{\sigma})$ is obtained from $\bar{R}$ by the adjunction of the zero $\bar{\sigma}$ of the irreducible polynomial $\bar{p}(x) \in \bar{R}[x]$ and hence $[S: R]=k[S: \bar{R}]$.

(9) Hereafter we shall refer to central algebraic (central transcendental) elements as algebraic (transcendental) elements. 
Next we shall prove

THEOREM 6.2. Let $S=R(\sigma)$ be a simple algebraic extension of $R$ where $[S: R]=k[S: \bar{R}]$. Then, $S$ is a principal extension of $R$ if and only if $k=1$. For any $k, N(S)=p(\sigma) R[\sigma]+N[\sigma]$ and hence there exists a positive integer $h$ such that $N(S)^{h}=0$.

Proof. Writing $q$ in the form stated in Theorem 6.1 we have, as in $\$ 4, N(q)$ $=p(x) R[x]+N(R[x])$. Since $R[x]$ is a principal extension of $R$ this can be written $N(q)=p(x) R[x]+N \cdot R[x]=p(x) R[x]+N[x]$. It follows from $2 \mathrm{~h}$ of [2] that $N(R[x] / q)=N(q) / q=(p(x) R[x]+N[x]) / q$. The isomorphism from $R[x] / q$ onto $S=R(\sigma)$ maps $(p(x) R[x]+N[x]) / q$ onto $p(\sigma) R[\sigma]+N[\sigma]$ and hence $N(S)=p(\sigma) R[\sigma]+N[\sigma]$. Now $N(q) / q$ is a nil ideal in $R[x] / q$ and, since the A.C.C. holds, the ideal $N(q)$ of $R$ is nilpotent modulo $q$. Thus there is a positive integer $h$ such that $N(S)^{h}=0$. Finally, $S$ is a principal extension of $R$ if and only if $p(\sigma) \in N[\sigma]$; i.e., if and only if $p(x) \in q^{\prime}$, where $q^{\prime}=p(x)^{k} R[x]+N[x]$. If $p(x) \in q^{\prime}$ then $q^{\prime}$ contains a regular polynomial of degree $D(\overline{p(x)})$. However, from $\S 3$, the minimal degree of the regular polynomials in $q^{\prime}$ is $D\left(\overline{\left.p(x)^{k}\right)}=k D(\overline{p(x)})\right.$. Hence $k D(\overline{p(x)}) \leqq D(\overline{p(x)})$ and therefore $k=1$. Conversely if $k=1$, the extension is clearly principal.

An element $\sigma$ of a ring $A$ is called principal with respect to a subring $R$ if $R(\sigma)$ is a principal extension of $R$. It follows from Theorem 6.2 that an algebraic element $\sigma$ is principal if and only if it is a root of a nontrivial fundamental irreducible $\left({ }^{10}\right)$ of $R[x]$.

EXAMPLe 6.1. Let $R$ be a completely $N$ primary ring satisfying the A.C.C. If $x$ is an indeterminate, the ring $R[x]$ is $N$ primary. Let $q$ be a regular, nontrivial $N$ primary ideal of $R[x]$ such that $q_{*}=0$. As above, we have that $q=\left(v(x) p(x)^{k}+n(x)\right) R[x]+N^{\prime}$ and $R[x] / q$ is a completely $N$ primary ring which contains $R$. Setting $\sigma=\bar{x}$, where $\bar{x}$ is the coset of $x$ in $R[x] / q$, then $\sigma$ is algebraic over $R$ with defining ideal equal to $q$.

EXAMPLE 6.2. Let $D$ be the division ring of quaternions with coefficients in the rational numbers and $D^{*}$ the division ring of quaternions with coefficients in the real numbers. For an indeterminate $x, R=D[x] /\left(x^{n}\right)$ is completely $N$ primary and is contained in the completely $N$ primary ring $R^{*}=D^{*}[x] /\left(x^{n}\right)$. If $\sigma=\sqrt{2}$, then $R_{1}=R(\sqrt{2})=D_{1}[x] /\left(x^{n}\right)$ where $D_{1}$ is the ring of quaternions with coefficients in the set of all real numbers of the form $a+b \sqrt{2}$ where $a$ and $b$ are rational numbers. Thus $R_{1}$ is a simple algebraic extension of $R$ of degree 2 .

For an indeterminate $y$, the ring $R[y]$ is $N$ primary. Then $y^{2}-2$ is a minimal degree polynominal satisfied by $\sqrt{2}$. One can use the division algorithm to show that the defining ideal of $\sqrt{2}$ is $q=\left(y^{2}-2\right) R[y]$. Then $q$ is $N$ primary and $R[y] / q \cong R_{1}$ where $R_{1}$ is an extension of $R$ of degree 2. Similarly, we could adjoin

(10) See $[2,2 e]$ for the meaning of this term. 
to $R_{1}$ the element $\sqrt{3}$. Thus $R_{2}=R_{1}(\sqrt{3})=D_{2}[x] /\left(x^{n}\right)$ where $D_{2}$ is the ring of quaternions with coefficients in the set of all real numbers of the form $a+b \sqrt{2}$ $+c \sqrt{3}+d \sqrt{6}$ where $a, b, c$ and $d$ are rational numbers. Then $R_{2}$ is an extension of $R_{1}$ of degree 2 and an extension of $R$ of degree 4 .

7. Quotient rings. If a ring $R$ has a right quotient ring as described in [5, p. 118] we shall denote this ring by $Q(R)$. Then $Q(R)$ is a ring containing $R$ such that every regular element of $R$ has an inverse in $Q(R)$ and any element of $Q(R)$ may be written in the form $a b^{-1}=a / b$ where $a, b \in R$ and $b$ is regular. A necessary and sufficient condition for the existence of $Q(R)$ is that for any pair of elements $a, b$ in $R, b$ regular, there exists a common right multiple $m=a b_{1}=b a_{1}$ such that $b_{1}$ is regular. We shall use this criterion to establish the following theorem, which generalizes a result of A. W. Goldie [4, p. 592].

THEOREM 7.1. Let $R$ be a ring with identity which satisfies the A.C.C. for right ideals and suppose the elements not in $N\left(R / N^{k}\right)$ are regular in $R / N^{k}$ for all positive integers $k$. Then $Q(R)$ exists.

Proof. Since the elements not in $N$ are regular in $R, R / N$ is an integral domain. Hence, if $N=0$, then by Theorem 1 of [4], $Q(R)$ exists. We proceed by induction on the smallest integer $n$ such that $N^{n}=0$. Assume that the theorem is true when $N^{k}=0$ and $N^{k-1} \neq 0$. In $R$ suppose $N^{k+1}=0$ and $N^{k} \neq 0$. Then the ring $\widetilde{R}=R / N^{k}$ satisfies the hypothesis of the theorem and, by the induction hypothesis, $Q(\tilde{R})$ exists.

Let $a \neq 0$ and $b \notin N$. If $a \in N^{k}$ we consider the right ideals $I_{n}=a R+b a R$ $+\ldots+b^{n} a R, n=0,1,2, \ldots$ By the A.C.C., $I_{t}=I_{t+1}$ for some integer $t$ and we may write $b^{t+1} a=a r_{0}+b a r_{1}+\ldots+b^{t} a r_{t}, r_{i} \in R$. Since $b^{t+1} a \neq 0$ and $N^{k+1}=0$, not all the $r_{i}$ are in $N$. Let $i=h$ be the first subscript for which $r_{i} \notin N$. It follows that $b^{t+1-h} a=a r_{h}+\ldots+b^{t-h} a r_{t}$ and hence $b\left(b^{t-h} a-a r_{h+1}-\ldots-b^{t-h-1} a r_{t}\right)$ $=a r_{h}$ where $r_{h}$ is regular. Thus $a$ and $b$ have a common right multiple. If, on the other hand, $a \notin N^{k}$, then, since $Q(\tilde{R})$ exists, we have $\tilde{a} \tilde{c}=\tilde{b} \tilde{d}$ in $\tilde{R}=R / N^{k}$ where $c \notin N$. Thus $a c=b d+e$ with $e \in N^{k}$. If $e=0$, we stop. Otherwise, as above, write $b f=e g$ where $g \notin N$, whence $a c g=b(d g+f)$ with $c g$ regular. Thus $Q(R)$ exists.

THEOREM 7.2. If $R$ is a completely $N$ primary ring which satisfies the A.C.C. for right ideals then $R[x]$ has a right quotient ring $Q(R[x])$.

Proof. From Theorem 1.3, for any integer $n$, the divisors of zero of $R / N^{n}[x]$ are contained in $N / N^{n}[x]$. Hence $R[x]$ satisfies the hypothesis of Theorem 7.1.

LEMMA 7.1. If a ring $R$ has a right quotient ring $Q(R)$ and if $R$ satisfies the A.C.C. for right ideals then $Q(R)$ satisfies the A.C.C. for right ideals.

Proof. Let $k$ and $h$ denote right ideals of $Q(R)$ where $k \subset h$. As in the proof of Lemma 1.3 of [3] it follows that $k_{*} \subset h_{*}$ in $R$. The lemma is now immediate. 
LEMMA 7.2. If a ring $R$ with A.C.C. on right ideals has a right quotient ring $Q(R)$ and if the elements not in $N$ are regular in $R$ then $Q(R)$ is completely $N$ primary.

Proof. Let $T=\{a / b \mid a / b \in Q(R), a \in N\}$. For any elements $a / b$ and $c / d$ of $T$, there exist regular elements $b_{1}$ and $d_{1}$ in $R$ such that $m=d b_{1}=b d_{1}$. Using the rule for addition in $Q(R)$ we have $a / b-c / d=\left(a d_{1}-c b_{1}\right) / m$ which is in $T$ since $a$ and $c$ are in $N$. Next, consider any elements $a / b$ of $T$ and $c / d$ of $Q(R)$. Let $c_{1}$, $b_{1} \in R, b_{1}$ regular, such that $c b_{1}=b c_{1}$. Then $(a / b)(c / d)=\left(a c_{1} / d b_{1}\right)$ which proves that $T Q(R) \subseteq T$. Similarly, one can prove that $Q(R) T \subseteq T$. Thus $T$ is an ideal in $Q(R)$. It is easily seen that for any positive integer $n$, the product of $n$ elements of $T$ can be written in the form $a / b$ where $a \in N^{n}$ and $b$ is regular. Since $N$ is nilpotent, $T \subseteq N(Q(R))$. Also, the elements of $Q(R)$ which are not in $T$ are units and hence $Q(R) / T$ is a division ring. Then $T$ is maximal ideal and $T=N(Q(R))$. Thus $Q(R)$ is completely $N$ primary.

LEMMA 7.3. If a ring $R$ has a right quotient ring $Q(R)$ which is completely $N$ primary then $Q(R)$ is the smallest completely $N$ primary ring containing $R$.

Proof. Let $R^{\prime}$ be a completely $N^{\prime}$ primary ring where $N^{\prime}=N\left(R^{\prime}\right)$ and suppose $R \subseteq R^{\prime}$. If $b \in R$ is regular then $b \notin N^{\prime}$ and hence $b$ has an inverse $b^{-1}$ in $R^{\prime}$. Thus $a, b \in R, b$ regular implies $a b^{-1} \in R^{\prime}$; that is, $Q(R) \subseteq R^{\prime}$.

THEOREM 7.3. Let $R$ be a completely $N$ primary ring which satisfies the A.C.C. for right ideals and let $q$ be a $P$ primary ideal of $R[x]$ with $q \subseteq N(R[x])$. Then $Q(R[x] / q)$ exists and is completely $N$ primary. Moreover, $Q(R[x] / q)$ satisfies the A.C.C. for right ideals and is the smallest completely $N$ primary ring containing $R[x] / q$.

Proof. We know, by Theorem 7.2, that $Q(R[x])$ exists. Furthermore, as in the proof of Lemma 1.2 of [3], one can show that $q=q^{*} \cap R[x]$, where $q^{*}$ is the extension of $q$ to $Q(R[x])$. It follows that the mapping $f(x)+q \rightarrow f(x)+q^{*}$, $f(x) \in R[x]$, is an isomorphism of $R[x] / q$ into $Q(R[x]) / q^{*}$. We shall identify $R[x] / q$ with the subring of $Q(R[x]) / q^{*}$ which corresponds to $R[x] / q$ under this isomorphism.

If $f(x)+q$ is regular in $R[x] / q$ then $f(x) \notin N[x]$. By Theorem $1.3, R[x]$ is $N[x]$ primary and consequently $f(x)$ is regular in $R[x]$. Hence $f(x)$ has an inverse $f(x)^{-1}$ in $Q(R[x])$ and we have $f(x)^{-1}+q^{*}=\left(f(x)+q^{*}\right)^{-1}$. Thus the regular elements of $R[x] / q$ have inverses in $Q(R[x]) / q^{*}$. Now let $f(x) g(x)^{-1}+q^{*} \in Q(R[x]) / q^{*}$ where $f(x), g(x) \in R[x], g(x)$ regular. Then $f(x) g(x)^{-1}+q^{*}=\left(f(x)+q^{*}\right)\left(g(x)+q^{*}\right)^{-1}$. This proves that $Q(R[x]) / q^{*}$ is a right quotient ring for $R[x] / q$.

The remaining part of the theorem follows from Lemmas 7.1, 7.2 and 7.3.

8. Simple transcendental extensions. In this section, $R$ will always denote a completely $N$ primary ring which satisfies the A.C.C. for right ideals and $A$ will denote a completely $N$ primary ring which contains $R$. 
Let $\sigma \in A$ be transcendental over $R$. Then $R[\sigma]$ is an $N$ primary ring where $N(R[\sigma])=N(A) \cap R[\sigma]$. For an indeterminate $x$ we have the usual homomorphism $\theta$ of $R[x]$ onto $R[\sigma]$ defined by $f(x) \theta=f(\sigma)$. The defining ideal $q$ of $\sigma$ is then a nil, $P$ primary ideal of $R[x]$ and $q_{*}=q \cap R=0$. Since $R[x]$ satisfies the A.C.C. for right ideals, $q$ is a nilpotent ideal and $q \subseteq N(R[x])=N[x]$ by $[2, \S 1]$. By Theorem 7.3, $Q(R[x] / q)$ exists and, since $R[\sigma] \cong R[x] / q, Q(R[\sigma])$ exists. Moreover, $Q(R[\sigma])$ is the smallest completely $N$ primary ring containing $R$ and $\sigma$. Hence $Q(R[\sigma])$ $=R(\sigma)$, the simple transcendental extension of $R$ by $\sigma$.Also, $R(\sigma)$ satisfies the A.C.C. for right ideals. This establishes the first part of

THEOREM 8.1. If $S=R(\sigma)$ is a simple transcendental extension of $R$ then $R(\sigma)=Q(R[\sigma])$ and $R(\sigma)$ satisfies the A.C.C. for right ideals. The division ring $(R(\sigma))^{-}=\bar{R}(\bar{\sigma})$ is obtained by adjoining the transcendental element $\bar{\sigma}$ to $\bar{R}$. To every unit of $S$ a unique order can be associated.

To prove the last part of the theorem we observe from above that $(R[\sigma])^{-}$ $\cong(R[x] / q)^{-} \cong(R / N)[x]=R[x]$, where $\bar{R}$ is a division ring. To every nonzero element $\bar{r}$ of $(R[\sigma])^{-}$a unique degree is associated, namely the degree of the polynomial of $\bar{R}[x]$ which is the image of $\bar{r}$ under the isomorphism from $(R[\sigma])^{-}$ onto $\bar{R}[x]$. If we extend this isomorphism to an isomorphism from $Q\left((R[\sigma])^{-}\right)$ onto $\bar{R}(x)=Q(\bar{R}[x])$ then to every nonzero element of $Q\left((R[\sigma])^{-}\right)$a unique degree is associated, namely the degree of the corresponding (image) element of $R(x)$. (The degree of a fraction, by definition, is the maximum of the degrees of the numerator and denominator.) Since $(Q(R[\sigma]))^{-} \cong Q\left((R[\sigma])^{-}\right)$there is a unique degree associated with each element of $(Q(R[\sigma]))^{-}$. Now the set of not nilpotent elements of the completely $N$ primary ring $Q(R[\sigma])=R(\sigma)$ coincides with the set of units of $Q(R[\sigma])$. We define the order of a unit $r$ of $R(\sigma)$ as the degree of the element $\bar{r}$ onto which $r$ is mapped by the natural homomorphism from $Q(R[\sigma])$ onto $(Q(R[\sigma]))^{-}$. Thus, to each unit of $Q(R[\sigma])=R(\sigma)$ a unique order is associ$\operatorname{ated}\left({ }^{11}\right)$.

THEOREM 8.2. Every completely $N$ primary ring $R$ which satisfies the A.C.C. for right ideals is properly contained in just such a ring. Specifically, $R \subset$ $Q(R[x])$, which is a completely $N$ primary ring satisfying the A.C.C. for right ideals.

Proof. See Theorem 7.2, Lemma 7.1 and Lemma 7.2.

LEMMA 8.1. Let $S$ be a principal extension of $R$ and let $N(R)=P(R)$ and $N(S)=P(S)$. Let $n$ be an ideal in $N(S)$ and suppose that $N(S / n)=N(S) / n$. Then $S / n$ is a principal extension of $R / n_{*}$, where $n_{*}=n \cap R$.

(11) Note that for $\sigma$ algebraic we have $R[\sigma]=R(\sigma)$ and hence in this case it is also true that $Q(R[\sigma])=R(\sigma)$. 
Proof. As in $[2, \S 2]$, we may assume that $\tilde{R}=R / n_{*} \subseteq S / n$ where, for any set $B \subseteq S, \widetilde{B}$ denotes the image of $B$ under the natural homomorphism from $S$ to $S / n$. Let $v$ be an element of $S$ such that $\tilde{v} \in N(S / n)=N(S) / n$. Then $v \in N(S)$ and, since $S$ is a principal extension of $R$ we may write $v=\sum v_{i} \sigma_{i}$ where $v_{i} \in N(R)$ and $\sigma_{i} \in S$. Hence $\tilde{v}=\sum \tilde{v}_{i} \tilde{\sigma}_{i}$ where $\tilde{v}_{i} \in N\left(R / n_{*}\right)$ and $\tilde{\sigma}_{i} \in S / n$. Thus $S / n$ is a principal extension of $R / n_{*}$.

THEOREM 8.3. If $S$ is a simple transcendental extension of $R$ then $S$ is a principal extension of $R$.

Proof. Let $S=R(\sigma)$ and let $n$ denote the defining ideal of the transcendental element $\sigma$. Applying Lemma 8.1 to $R[x]$ and $R$, and noting that $n_{*}=n \cap R=0$, we have that $R[x] / n$ is a principal extension of $R / n_{*}=R$. Thus $R[\sigma]=R[x] / n$ is a principal extension of $R$. Now $Q=Q(R[\sigma])$ is a principal extension of $R[\sigma]$ since $N(Q)$ consists of elements of the form $a / b=a b^{-1}$ where $a \in N(R[\sigma])$ and $b^{-1} \in Q(R[\sigma])$. Hence $N(Q)=N(R[\sigma]) \cdot Q(R[\sigma])=N(R) \cdot R[\sigma] \cdot R(\sigma)=N(R) \cdot R(\sigma)$, i.e., $Q(R[\sigma])=R(\sigma)$ is a principal extension of $R$.

EXAMPLE 8.1. Let $R$ be a completely $N$ primary ring which satisfies the A.C.C. for right ideals. Let $x$ be an indeterminate and let $n$ be any nil, $P$ primary ideal of $R[x]$ such that $n_{*}=0$. Setting $\sigma=\bar{x}$, where $\bar{x}$ is the coset of $x$ in $R[x] / n$, then $\sigma$ is transcendental over $R$ with defining ideal $n$.

EXAMPLE 8.2. Let $R$ and $R^{*}$ be as in Example 6.2 and let $F$ be the field of rational numbers. Then for the transcendental number $\pi$ we have $R(\pi)=D^{\prime}[x] /\left(x^{n}\right)$ where $D^{\prime}$ is the ring of quaternions with coefficients of the form $p(\pi) / q(\pi)$ where $p(\pi)$ and $q(\pi)$ are elements of $F[\pi], q(\pi) \neq 0$.

\section{BIBLIOGRAPHY}

1. E. H. Feller, The lattice of submodules of a module over a noncommutative ring, Trans. Amer. Math. Soc. 81 (1956), 342-357. 79-91.

2. - Properties of primary noncommutative rings, Trans. Amer. Math. Soc. 89 (1958),

3. E. H. Feller and E. W. Swokowski, Reflective $N$-prime rings with the ascending chain condition, Trans. Amer. Math. Soc. 99 (1961), 264-271.

4. A. W. Goldie, The structure of prime rings under ascending chain conditions, Proc. London Math. Soc. 8 (1958), 589-608. 1943.

5. N. Jacobson, The theory of rings, Math. Surveys, No. 2, Amer. Math. Soc., New York,

6. - Structure of rings, Amer. Math. Soc. Colloq. Publ. Vol. 37, Amer. Math. Soc. New York, 1956.

7. E. Snapper, Completely primary rings. I, Ann. of Math. (2) 52 (1950), 666-693.

8. - Completely primary rings. II, Ann. of Math. (2) 53 (1951), 125-142.

UNIVERSITY OF WISCONSIN-MILWAUKEE, MilWAUKeE, Wisconsin

MARQUETTE UNIVERSITY, MilWAUKeE, WiSCONSIN 